\title{
ACTIVE RELEASE OF MATERIAL FROM RAT BLASTOCYSTS DEVELOPING IN VITRO
}

\author{
V. BITTON-CASIMIRI, J. L. BRUN AND A. PSYCHOYOS \\ I.N.S.E.R.M., Hôpital de Bicêtre, 94-Bicêtre, France
}

(Received 21st Fune 1971)

\begin{abstract}
Summary. Rat blastocysts, flushed from the uterus at the moment of the initiation of the implantation process and observed in vitro by timelapse cinematography, were seen to undergo a series of contractions and dilatations. By these movements, vesicles, containing granular material, emerged through the zona pellucida into the culture medium.
\end{abstract}

In all species so far studied, the earliest known step in the series of interactions between the ovum and the endometrium which lead to implantation, is an increase in the permeability of the endometrial capillaries surrounding the blastocysts. In the rat, this early response of the sensitive endometrium to the presence of the blastocyst is manifested on the afternoon of the 5th day of pregnancy. As the volume of the blastocoele is considerably reduced in most of the blastocysts recovered from the uterus at this moment, it has been suggested that components of the fluid released from the blastocoele may constitute the stimulus for inducing the vascular changes in the endometrium (Psychoyos, 1967).

In this paper, we report observations by time-lapse cinematography on the behaviour of blastocysts obtained from Wistar rats at noon on the 5th day of pregnancy (106 hr after the estimated time of ovulation) by flushing the uterine lumen with Tyrode. The culture medium was Eagle L medium containing $5 \%$ of foetal calf serum (DIFCO). After being rinsed three times in this medium, blastocysts were picked up in groups of four to five in a small drop of medium and were placed under paraffin oil in a plastic tissue culture dish; the culture dish was then placed under a microscope kept in an ambient temperature of $37^{\circ} \mathrm{C}$ and in a humid atmosphere containing $5 \% \mathrm{CO}_{2}$ in air (Bitton-Casimiri \& Psychoyos, 1968). A film at the rate of one frame per minute was taken, using a $16 \mathrm{~mm}$ Paillard Bolex camera, adapted to a time-lapse photographic apparatus (Wild).

When recovered from the uterus, the blastocysts were still surrounded by an apparently intact zona pellucida. From the beginning of their life in vitro, they were seen to undergo a series of dilatations and contractions. These movements lasted for a period of about $48 \mathrm{hr}$, during which the zona pellucida ruptured and the blastocysts abandoning it started to dilate. During phases of dilatation, the volume of the blastocoele increased progressively over 5 to $6 \mathrm{hr}$, until the whole blastocyst reached a maximum size of about $150 \mu \mathrm{m}$ diameter. A sudden, strong contraction, completed in less than $60 \mathrm{sec}$, then occurred, reducing the 
blastocyst to a small dark mass of about $70 \mu \mathrm{m}$ diameter at the centre of the distended zona. With the first contractions, one large vesicle, or several smaller ones of about $20 \mu \mathrm{m}$ diameter, could be seen on the surface of the blastocyst, in the space between it and the zona pellucida. As the blastocyst resumed the dilatation phase, these vesicles were pushed towards the zona pellucida and compressed against its inner surface. Apparently, the zona ruptures at the point of the contact with the vesicle since it is from this point that the vesicle finally emerges into the culture medium under the pressure of the dilatated blastocyst. Later, the granular material contained in the vesicle is dispersed, leaving what seems to be an emptied, thin layer (Plate 1).

The occurrence of rhythmic cycles of contraction and dilatation has already been recorded for mouse (Cole \& Paul, 1965) and rat (Bitton-Casimiri, Brun \& Psychoyos, 1970) blastocysts developing in vitro and has been associated with the escape of the embryo from the zona pellucida. The observations reported here not only confirm that these movements definitely facilitate such an escape but also show that they induce the release and expulsion of material from the blastocyst. Presumably, the released material in vivo could be promptly taken up by the luminal epithelium which, at the moment of the optimal uterine sensitivity, shows highly absorbtive activity (Vokaer \& Leroy, 1962). Components of this material could well be the stimulus for the endometrial response to the blastocyst.

This work was supported by a grant from the Population Council, New York.

\section{REFERENCES}

Bitton-Gasimiri, V., Brun, J. L. \& Psychoyos, A. (1970) Comportement in vitro des blastocystes du 5 jour de la gestation chez la ratte. Etude micro-cinematographique. C. r. hebd. Seanc. Acad. Sci., Paris, 270, 2979.

Bitton-Casimiri, V. \& Psychoyos, A. (1968) Développement du blastocyste du rat in vitro. C. $r$. hebd. Séanc. Acad. Sci., Paris, 267, 762.

Cole, R. J. \& PAUL, J. (1965) Properties of cultured pre-implantation mouse and rabbit embryos and cell strains derived from them. In: Preimplantation Stages of Pregnancy, p. 82. Ciba Fdn Symp. Churchill, London.

Psychoуоs, A. (1967) The hormonal interplay controlling egg-implantation in the rat. Adv. Reprod. Physiol. 2, 257.

VoKAer, R. \& Leroy, F. (1962) Experimental study on local factors in the process of ova implantation in the rat. Am. 7. Obstet. Gynec. 83, 141. 

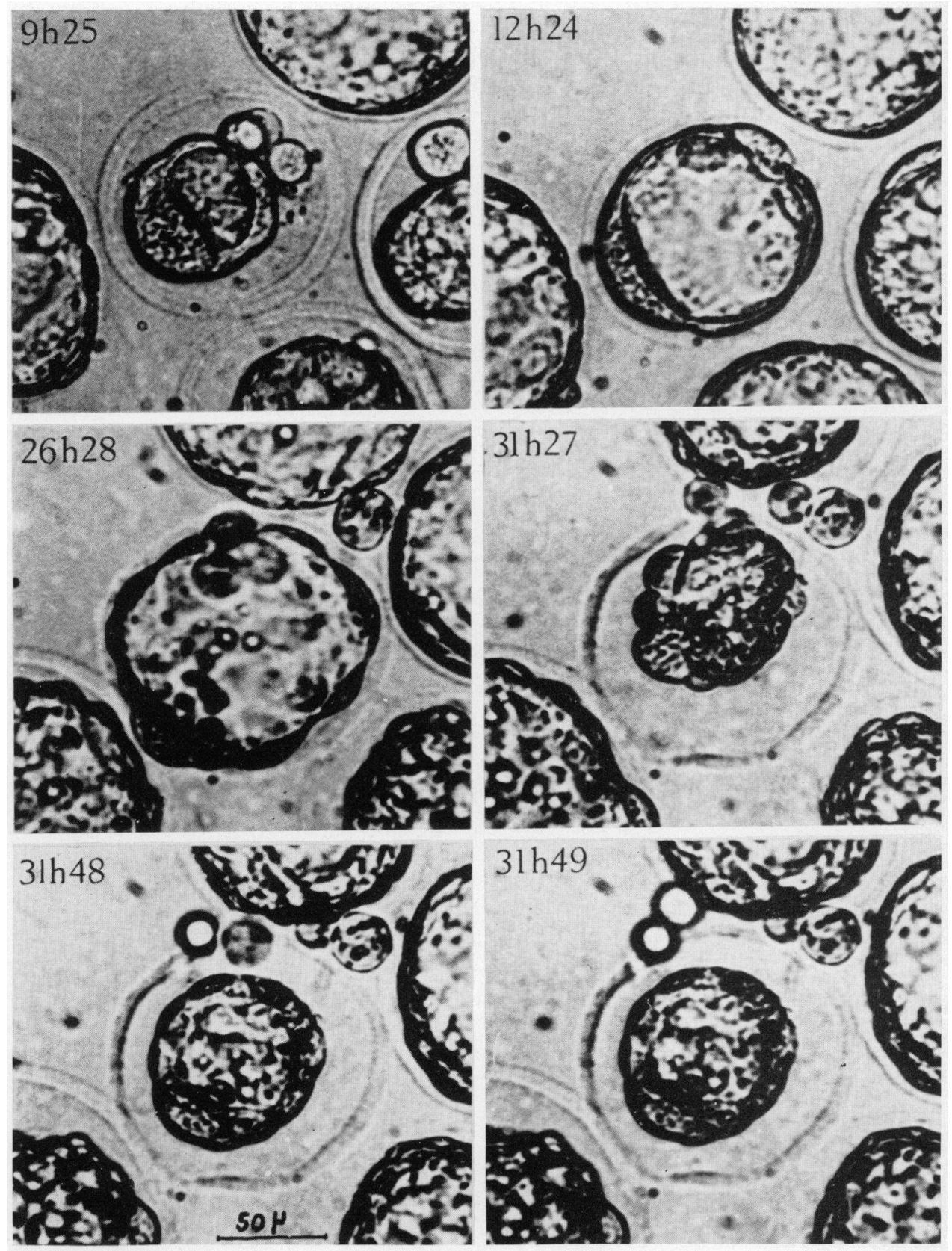

From a time-lapse film of rat blastocysts on the 5th day of pregnancy. Three vesicten are expelled from the blastocysts of the centre of the picture at its first contraction $(9 \mathrm{hr} 25$ min). Note the abrupt emptying of these vesicles. 